\title{
The Multicell Volume of Fluid (MC-VOF) Method for the Free Surface Simulation of MFD Flows. Part I: Mathematical Model
}

\author{
Ovidiu PEŞTEANU and Egbert BAAKE
}

Institute of Electrotechnology, Leibniz University of Hanover, Wilhelm-Busch-Str. 4, D-30167 Hanover, Germany. E-mail: pesteanu@etp.uni-hannover.de

(Received on November 1, 2010; accepted on February 17, 2011)

\begin{abstract}
This paper is the first part of a two-part paper which presents a simulation algorithm of unsteady, electromagnetically driven molten metal flows with free surfaces. At the free boundary, the variable space-distribution of the normal Lorentz forces is taken into account by proper computation of the electromagnetic field and pressure. For each calculation time step, a transport equation of the melt's volume is solved for multicell blocks and subsequently, the free surface is reconstructed by an inward gathering of the melt volume. Therefore, the free surface can be more accurately simulated with the following improvements:

(1) Consideration of the normal electromagnetic force densities exerted on the melt surface.

(2) Strictly volume conserving displacement of the free surface.

(3) Absence of numerically created holes in the melt or of separated fluid droplets, respectively.

Comparisons between computational and experimental results to verify the validity of the mathematical model will be presented in the second part of the paper.
\end{abstract}

KEY WORDS: magneto-fluid dynamics; finite difference method; unsteady turbulent flow; pressure calculation in electromagnetic field; free surface simulation; fluid volume conservation.

\section{Introduction}

One of the most widely used methods for the simulation of unsteady free surfaces is the Volume of Fluid (VOF) method $^{1-7)}$ based on the advection equation of a VOF function, $F$, indicating the fractional volume of a computational cell filled with fluid, ${ }^{1)}$ i.e. $F=1$ in the completely filled fluid (F) cells (Fig. 1), $0<F<1$ in the partially filled surface (S) cells and $F=0$ in the empty (E) cells, respectively.

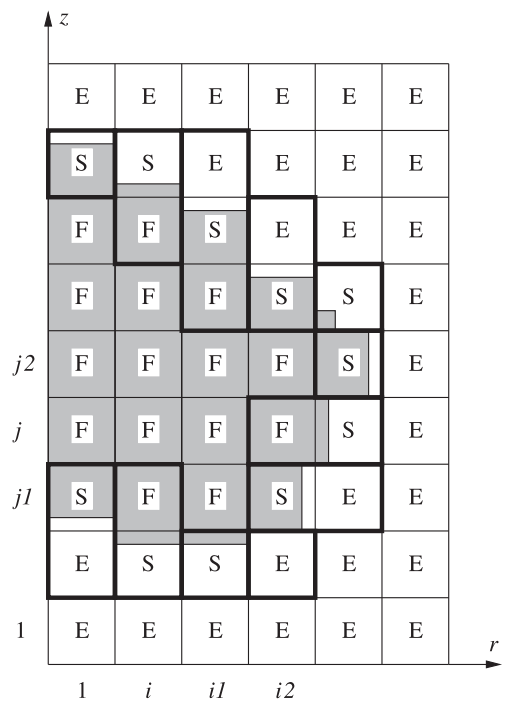

Fig. 1. Cell labeling and thick line cell blocks for applying the MCVOF method.
After every calculation time step, the free surface is commonly approximated either by a Simple Line Interface Construction (SLIC) using lines or planes aligned with the coordinate axes, ${ }^{1-3,5-7)}$ or by the more accurate Piecewise Linear Interface Construction (PLIC) employing inclined lines or oblique planes. ${ }^{2,4-7)}$

The pressure in an $\mathrm{S}$ cell is calculated as a linear inter- or extrapolation between the pressure in an adjacent $\mathrm{F}$ cell and the pressure at the free surface. ${ }^{1)}$ The thus obtained values are accurate for the hydrodynamic flows driven by constant force densities, but could be inaccurate for Magneto-Fluid Dynamic (MFD) flows driven by variable electromagnetic force densities, increasing toward the free surfaces of the molten metals. Therefore, in this paper a proper calculation of the near-surface pressure will be established for MFD flows.

The simulations with the donor-acceptor method ${ }^{1)}$ and the briefly mentioned surface reconstruction methods can yield values $F<0$ or $F>1$ and by their rounding to 0 and 1 , respectively, the volume conservation of an incompressible fluid is annihilated. Another drawback of these methods is the numerical creation of unphysical holes in the fluid or of 'flotsam' and 'jetsam', which are small droplets separated from the free surface. ${ }^{2,3,5-7)}$

Even if the Adams-Bashforth method is applied to integrate the advection equation of $F$, a blurred liquid-gas interface results in the modeling of a two-phase flow and to make it sharp, an anti-diffusion correction of the $F$-values must be introduced after each calculation time step. ${ }^{8)}$ Likewise even if a third-order upwind scheme is used for the 
advection equation of an index function $\Phi$ employed instead of $F^{9,10)}$ the sharpness of the interface is gradually lost during the computation and values of $\Phi$ outside of its definition domain result, which must be permanently rounded to the nearest admissible boundary value. To keep the interface thin, proper non-linear functions of $\Phi$ must be introduced for the physical properties within the transitional interface. ${ }^{9,10)}$

For a rigorous volume conserving and to determine a well-defined free surface contour without the generation of unrealistic holes or droplets, the VOF method was combined in Cartesian coordinates with the advection of a Local Height Function (LHF), ${ }^{3)}$ and successfully employed for the simulation of the laminar flows in a partially water filled spacecraft, ${ }^{5)}$ for wave impact problems and at water entry of free falling objects, ${ }^{6,7)}$ respectively.

Owing to its calculation stability, simplicity and robustness as well as its experimentally verified accuracy, the VOF-LHF method was found to be the most suitable for the development of a numerical algorithm to model MFD free surface flows, but in applying this efficient method also for axisymmetric flows, the usage of an LHF in radial direction is relatively complicated, because the grid cells have different volumes depending on their radii. Other difficulties can appear e.g. at the calculation of the closed free surfaces of inductively levitated melts, by the overlapping of either: 1) two reciprocally normal LHFs opposite to a surface convex corner, where the surface orientation changes from a more horizontal to a more vertical one, or 2) two vertical LHFs when the corresponding upper and lower horizontal parts of an unsteady free surface become very near to each other. In both cases the possibility exists of multiple fluid fluxing hindering the conservative melt volume advection.

Owing to these difficulties, in this paper the VOF-LHF method is modified by applying the VOF method for the fluid in non-overlapping Multi-Cell (MC) blocks located at the free surface (Fig. 1) and a strictly volume conserving surface reconstruction using the SLIC method is adapted to this $\mathrm{MC}$ algorithm. The main objective of the paper is extending the developed MC-VOF method to an accurate simulation also of turbulent MFD flows by using the Finite Difference (FD) method for a staggered grid and the $k-\varepsilon$ model, respectively.

\section{Calculation of the Electromagnetic Field}

The sinusoidal electromagnetic field is determined by solving the equation of the magnetic vector potential, $\mathbf{A}$,

$$
\Delta \underline{\mathbf{A}}-j \omega \mu_{0} \sigma \underline{\mathbf{A}}=\mu_{0} \sigma(-\underline{\mathbf{E}}+\nabla \underline{V})-\mu_{0} \underline{\mathbf{J}}_{\text {ind }}, \quad j=\sqrt{-1}
$$

where, the complex representations of the sinusoidal physical quantities are underlined, $\omega$ and $\sigma$ are the net angular frequency and the electrical conductivity, respectively, $\underline{\mathbf{E}}=\mathbf{u} \times \underline{\mathbf{B}}$ denotes the electric field intensity due to the motion with the velocity $\mathbf{u}$ and $\underline{\mathbf{J}}_{\text {ind }}$ represents the density of the source currents, e.g. in an inductor coil.

The electric scalar potential, $V$, is calculated inside the melt as solution of the equation

$$
\nabla \cdot(\sigma \nabla \underline{V})=\nabla \cdot(\sigma \underline{\mathbf{E}})-j \omega \nabla \cdot(\sigma \underline{\mathbf{A}})
$$

and is used to annul the normal eddy current density at the conductor surface.

Equations (1) and (2) are numerically solved by considering all $\mathrm{S}$ cells as being completely filled. ${ }^{11)}$ Thus, both the electromagnetic and force fields are computed in the $S$ cells employing the electrical conductivity of the melt. The use for the $\mathrm{S}$ cells of a reduced conductivity, e.g. $F \sigma<\sigma{ }^{12)}$ yields smaller Lorentz forces driving unrealistic, too weak near-surface tangential flows, and as a result, a wrong free surface form can be calculated.

To reduce the computational effort, the magnetic field can be determined in the regions free of eddy currents by the magnetic scalar potential, $\psi$, when an $\mathbf{A}, V-\psi$ formulation is used in the field calculation. ${ }^{13)}$ For well-known electromagnetic fields, analytical expressions will be used for the Lorentz force components, e.g. for the stirring in a rotational two-pole magnetic field. ${ }^{14)}$ More details of the electromagnetic calculation are given in the second part of the paper, for the different laboratory setups used for the experimental validation.

\section{Calculation of Turbulent Flow}

The general transport equation governing the melt flow when using the $k-\varepsilon$ turbulence model can be written as

$$
\frac{\partial \Phi}{\partial t}+\nabla \cdot(\mathbf{u} \Phi)=\nabla \cdot\left(v_{\text {eff } \Phi} \nabla \Phi\right)+\frac{S_{\Phi}}{\rho},
$$

in which, $\Phi$ represents a velocity component (for the momentum equations), the kinetic energy of turbulence, $k$, or its rate of dissipation, $\varepsilon, v_{\text {eff }} \Phi$ denotes the effective viscosity for the transported variable $\Phi, \rho$ is the density and the source term $S_{\Phi}$ comprises the resulting force density and the pressure gradients in the momentum equations and the production and destruction terms for $k$ and $\varepsilon$ in the turbulence equations, respectively.

The explicitly discretized general transport Eq. (3) can be written for a point $P$ of the staggered grid as

$$
\Phi_{P}^{\text {new }}-\Phi_{P}=\Delta t\left(\sum a_{\mathrm{nb}} \Phi_{\mathrm{nb}}-a_{P} \Phi_{P}+T\right)
$$

where the superscript 'new' indicates a new value, the summation is performed over all neighbor grid points of $P$ denoted by the subscript ' $n b$ ' and $T$ is the FD approximation of all other terms. The time-related evolution of the flow is numerically simulated by using for all explicitly discretized momentum and turbulence field equations the stability limited time step deduced by applying Brauer's theorem ${ }^{15}$ )

$$
\Delta t=\frac{C_{\mathrm{s}}}{\max \left\{a_{P}\right\}}
$$

in which $\max \{\}$ terms the greatest value in all discretized transport equations from the last time level and $C_{\mathrm{s}} \leq 1$ is a safety factor to avoid instabilities due to nonlinearity, interlinkage, variable source terms and the third-order upwind scheme QUICK ${ }^{16)}$ used for the convective terms in the momentum equations.

For each calculation time step, the FF, FS and SS velocities are first determined by the discretized momentum equations $^{5-7)}$ and subsequently, the SOLution Algorithm (SOLA $)^{1,17)}$ is employed for the iterative correction of pressure and velocity fields to satisfy continuity. As the velocity 
and pressure are determined in S cells by the boundary conditions presented in the next section, the iterative SOLA code is used only for the F cells. ${ }^{11)}$ Finally, $k$ and $\varepsilon$ are computed in all $\mathrm{F}$ and $\mathrm{S}$ cells by considering all $\mathrm{S}$ cells to be also completely filled. ${ }^{11)}$

On a solid boundary, e.g. the crucible wall of an induction furnace, the wall shear stress is calculated by the friction velocity from the logarithmic law of the near-wall tangential velocity, ${ }^{14,18)}$ a vanishing normal gradient is imposed for $k$ and a boundary value obtained assuming local equilibrium of turbulence is employed for $\varepsilon^{14,16,18)}$

\section{Free Surface Boundary Conditions}

On the outer faces of the $\mathrm{S}$ cells, the normal eddy current densities can be annulled by the electric potential $V$.

For the flow calculation, a free surface reconstructed by the SLIC method, e.g. of a levitated melt (Fig. 1), can be divided into a first lower More Horizontal Section (MHS) consisting of $\mathrm{S}$ cells with $1 \leq i \leq i 1$, in which the surface is approximated by horizontal line segments, a second More Vertical Section (MVS), in the S cells of which, with $j 1 \leq j \leq$ $j 2$ the surface consists of vertical line segments, a corner $\mathrm{S}$ cell $(i 2+1, j 2+1)$, where the surface is reconstructed by a square, and a last upper MHS consisting of $\mathrm{S}$ cells with $1 \leq$ $i \leq i 2$, respectively.

The boundary conditions will be presented for an axisymmetric flow with the velocity components, $u$ and $v$, along the $r$ - and $z$-directions. The tangential and normal stress conditions at the free surface can be written as ${ }^{5-7)}$

$$
\frac{\partial u}{\partial z}+\frac{\partial v}{\partial r}=0, \quad p_{\mathrm{s}}=p_{\mathrm{a}}+p_{\gamma}+2 \mu_{\mathrm{eff}} \frac{\partial u_{n}}{\partial n}, \quad p_{\gamma}=-\gamma \kappa \ldots
$$

where, $p_{\mathrm{s}}$ is the pressure at the free surface, $p_{\mathrm{a}}$ indicates the ambient pressure, $p_{\gamma}$ denotes the pressure due to the surface tension $\gamma,{ }^{19)} \mu_{\text {eff }}$ represents the effective viscosity, the rightmost derivative of the normal velocity, $u_{n}$, is calculated along the normal direction to the free surface and $\kappa$ is the surface mean curvature. For the S cells of an MHS and simplified also for the corner $\mathrm{S}$ cells, $u_{n}=v$, while for the $\mathrm{S}$ cells belonging to an MVS $u_{n}=u$.

\subsection{Velocity, Turbulent Energy and Dissipation at the Free Surface}

In all $\mathrm{S}$ cells the continuity equation

$$
\frac{1}{r} \frac{\partial}{\partial r}(r u)+\frac{\partial v}{\partial z}=0
$$

is employed because of the following reasons:

- If instead of Eq. (7) another condition is used, e.g. a velocity extrapolation, ${ }^{6,7)}$ then, when an $\mathrm{S}$ cell where $\operatorname{div} \mathbf{u} \neq 0$ changes to an $\mathrm{F}$ cell with $\operatorname{div} \mathbf{u}=0$, numerical, nonrealistic spikes occur in the pressure field. ${ }^{7}$

- When using the $k-\varepsilon$ (or the Reynolds stress) model, the production terms are physically more accurately computed in those $\mathrm{S}$ cells, where the continuity equation was used. ${ }^{11)}$

Equation (7) can be applied immediately for the middle $\mathrm{S}$ cell shown in Fig. 2(a), where the SE velocity, $v_{n}$, follows from the other three velocities calculated by the momentum equations. Subsequently, the upper EE velocity, $u_{\mathrm{EE}}$, is determined from the FD approximation of the tangential stress condition (6). For an $\mathrm{S}$ cell with two open sides not positioned opposite each other (Fig. 2(b)), the two SE velocities, $u_{e}$ and $v_{n}$, are computed either by applying Eq. (7) only in the $r$-and only in the $z$-direction, respectively: $r_{e} u_{e}=$ $r_{w} u_{w}$ and $v_{n}=v_{s}$, or, in case of a secondary flow, e.g. the flow driven by the centrifugal forces in the rotational stirring, by setting more simple $u_{e}=0$ and calculating $v_{n}$ from the discretized Eq. (7). Other SE and EE velocities needed for solving the momentum equations and calculation of the production terms are determined by the boundary conditions given in Ref. 20) for the simulations with the Marker and Cell method.

Through the outer surfaces of the S cells, $k$ and $\varepsilon$ are neither convected nor diffused and consequently, the SE velocities are set to zero for their convective terms in contrast to the calculation of the production terms.

\subsection{Pressure at the Free Surface}

In applying the FD method, the variable potential component, $f_{\mathrm{p}}$, of the resulting vertical force density in an electromagnetic field can be assumed to be piecewise constant, e.g. for an S cell of a MHS, $f_{\mathrm{p}}=f_{\mathrm{FS}}$ in Fig. 3(a) between the central points of the neighboring $\mathrm{F}$ and $\mathrm{S}$ cells shown in Fig. 3(b). If the free surface lies between these central points, then the pressures at the free surface and in the $\mathrm{S}$ cell can be written as

$$
p_{\mathrm{s}}=p_{\mathrm{F}}+f_{\mathrm{FS}} d, \quad p_{\mathrm{S}}=p_{\mathrm{F}}+f_{\mathrm{FS}} h
$$

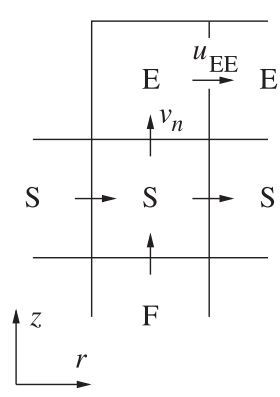

(a)

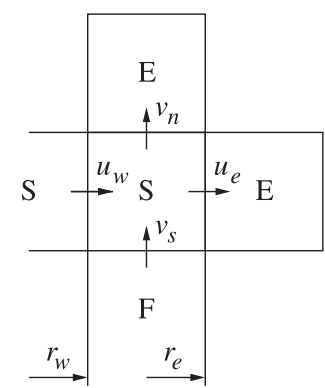

(b)
Fig. 2. Concerning the calculation of (a) one and (b) two SE velocities.

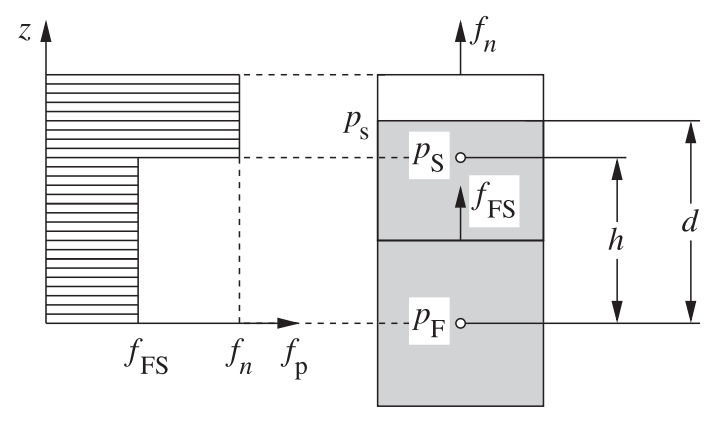

(a)

(b)

Fig. 3. Concerning the pressure calculation (a) for a piecewise constant distribution of the potential force density (b) in an S cell on a MHS of the free surface. 
and after elimination of $f_{\mathrm{FS}}$, one obtains the known extrapolated pressure ${ }^{1)}$

$$
p_{\mathrm{S}}=\eta p_{\mathrm{s}}+(1-\eta) p_{\mathrm{F}} \text { if } d \leq h, \quad \eta=h / d
$$

For $d>h$, the $\mathrm{S}$ cell pressure can be more accurately calculated by the resulting normal force density, $f_{n}$, acting on the outer face of the cell (Fig. 3(b)), which is not used in the discretized momentum equations ${ }^{11)}$

$$
p_{\mathrm{S}}=p_{\mathrm{s}}-f_{n}(d-h) \text { if } d>h
$$

For the S cells of an MVS, Eqs. (9) and (10) are applied in the $r$-direction, while for a corner $\mathrm{S}$ cell with an VOF function $F_{\mathrm{S}}, p_{\mathrm{S}}$ can be computed in a simplified manner the same as for an MHS by using the already calculated pressure from the adjacent $\mathrm{S}$ cell in $z$-direction instead of $p_{\mathrm{F}}$ and $d=\left(0.5+F_{\mathrm{S}}\right) h$, respectively.

\section{Free Surface Treatment}

\subsection{Free Surface Displacement}

Each MC block is composed of several grid cells or of only one cell and contains either one single $\mathrm{S}$ cell or an $\mathrm{E}$ cell located in a concave corner of the free surface, respectively (Fig. 1). For example, the block corresponding to an $\mathrm{S}$ cell, $(i, j)$, on an MHS shown in Fig. 4(a) comprises the cells of a column, $i$, from the lowest, $(i, j a)$, to the uppermost cell, $(i, j b)$. The two limits, $j a$ and $j b$, are chosen in such a way that between them, each row of the cells, $i-1, i$ and $i+$ 1, contains at least an S cell which belongs to the MHS or is a corner $\mathrm{S}$ cell, respectively.

On an MVS, a radial MC block corresponding to an $\mathrm{S}$ cell $(i, j)$ (Fig. 4(b)) comprises the cells of a row, $j$, from an innermost cell, $(i a, j)$, to an outermost grid cell, $(i b, j), i a$ and $i b$ being defined in $r$-direction in a similar manner as $j a$ and $j b$ in $z$-direction. For a simple application of the VOF method, the radial MC block is more suitable compared to a radial LHF, because the LHF must be introduced by a radiusaveraging of the VOF fractions ${ }^{11)}$ and requires for the free surface reconstruction a relatively complicated, radiusdepending calculation of the individual VOF functions from the LHF-value. Between an MHS and a neighboring MVS, the MC block is composed of only one single cell, which can be partly filled (Fig. 5(a)) or empty (Fig. 5(b)), respec-

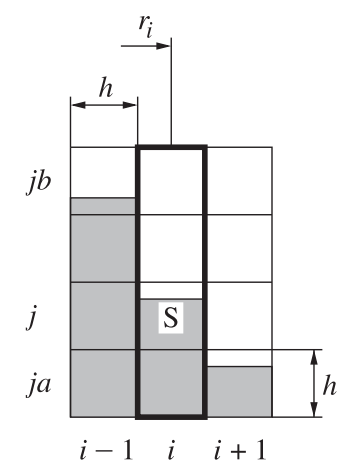

(a)

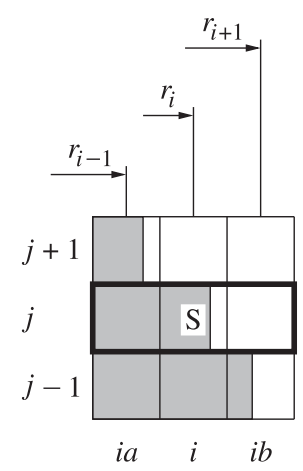

(b)
Fig. 4. Thick line MC blocks on (a) an MHS and (b) an MVS of the free surface. tively.

For each of the presented MC blocks corresponding to an $\mathrm{S}$ or $\mathrm{E}$ cell $(i, j)$, the conservation equation of the melt volume during a time step $\Delta t$ can be expressed, when using an uniform grid of size $h$, as

$$
\left(C_{i, j}^{\text {new }}-F_{i, j}\right) V_{i}+Q_{\text {block }} \Delta t=0
$$

in which, $C_{i, j}$ represents a preliminary VOF-type function of the block's grid cell $(i, j)$, the superscript 'new' indicates a new calculated value, $V_{i}=2 \pi r_{i} h^{2}$ is the volume corresponding to the cell $(i, j)$ and $Q_{\text {block }}$ denotes the algebraic sum of the outward flow rates determined through all these faces of the block cells, which lie on the block surface. For example, the radial outward flow rate, $Q_{e}$, across the eastern face of a cell, $(i, m)$, shown in Fig. 6(a), with a radius, $r_{e}$, and belonging to the vertical MC block of a MHS (Fig. 4(a)), is given by

$$
Q_{e}=2 \pi r_{e} h u_{e} F_{e}
$$

where the upwinded VOF function, $F_{e}$, is set to $F_{e}=F_{i, m}$, if the outgoing velocity, $u_{e}$, is positive. If $u_{e}<0$, then $F_{e}=$ $F_{i+1, m}$ when the cell $(i+1, m)$ is an $\mathrm{F}, \mathrm{E}$ or an $\mathrm{S}$ cell on an MHS (Fig. 6(a)), $F_{e}=1$ if the eastern cell is an $\mathrm{S}$ cell on an MVS (Fig. 6(b)) and $F_{e}=\sqrt{F_{i+1, \mathrm{~m}}}$ when the cell $(i+1, m)$ is a corner $\mathrm{S}$ cell $($ Fig. $6(\mathrm{c}))$, respectively.

\subsection{Free Surface Reconstruction}

The new value, $C_{i, j}^{\text {new }}$, obtained for each MC block is conservatively redistributed as the new VOF functions $F^{\text {new }}$ of the block cell $(i, j)$ and its adjacent cells, as follows. For example, if for the $\mathrm{S}$ cell of the radial MC block on an MVS (Fig. 4(b)), $0 \leq C_{i, j}^{\text {new }} \leq 1$, then its VOF function is updated to the new time level as $F_{i, j}^{\text {new }}=C_{i, j}^{\text {new }}$. For $C_{i, j}^{\text {new }}<0$, the cell $(i$,

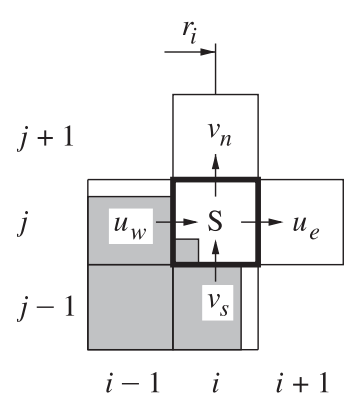

(a)

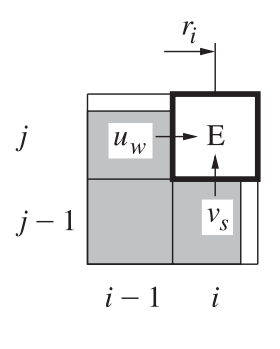

(b)
Fig. 5. Thick line single cells containing either (a) a corner $\mathrm{S}$ cell or (b) an E cell in a concave corner of the free surface.

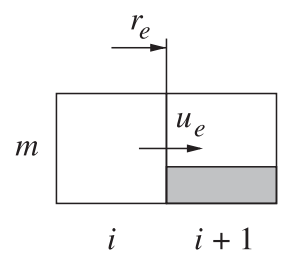

(a)

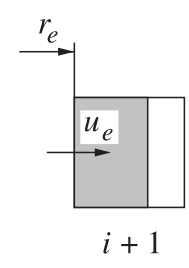

(b)

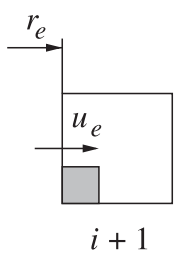

(c)
Fig. 6. Concerning the flow rate calculation through the eastern side of a cell, $(i, m)$, belonging to a vertical MC block. 
$j$ ) will be emptied and the melt volume is inward gathered according to

$$
F_{i, j}^{\text {new }}=0, \quad F_{i-1, j}^{\text {new }}=F_{i-1, j}+\frac{r_{i}}{r_{i-1}} C_{i, j}^{\text {new }}
$$

thus preventing the creation of artificial holes or subgridsize bits separated from the mean melt body. If $C_{i, j}^{\text {new }}>1$, then cell $(i, j)$ is completely filled and the volume excess is transferred in the direction normal to the MVS to its eastern empty cell:

$$
F_{i, j}^{\text {new }}=1, \quad F_{i+1, j}^{\text {new }}=\frac{r_{i}}{r_{i+1}}\left(C_{i, j}^{\text {new }}-1\right)
$$

For the newly created $\mathrm{S}$ cell $(i+1, j)$, the values of $k, \varepsilon$ and $\mu_{\text {eff }}$ are taken from its neighboring filled cell $(i, j)^{11)}$

$$
\Phi_{i+1, j}^{\text {new }}=\Phi_{i, j}, \quad \Phi=k, \varepsilon, \mu_{\text {eff }}
$$

The ratios of radii in Eqs. (13) and (14) consider the volume modifying of the grid cells in $r$-direction.

In vertical MC blocks, the new $F$ - and $\Phi$-values are assigned using formulae analogous to Eqs. (13)-(15), but without the radii ratios, because the grid cells of a column have the same volumes in $z$-direction, e.g. if for the $\mathrm{S}$ cell in Fig. 4 (a) $C_{i, j}^{\text {new }}<0$, then cell $(i, j)$ will be emptied and the melt volume is inward gathered according to

$$
F_{i, j}^{\text {new }}=0, \quad F_{i, j-1}^{\text {new }}=F_{i, j-1}+C_{i, j}^{\text {new }}
$$

In case of the thick circumlined $\mathrm{S}$ cell shown in Fig. 5(a), if $0 \leq C_{i, j}^{\text {new }} \leq 1$, then $F_{i, j}^{\text {new }}=C_{i, j}^{\text {new }}$ too. For $C_{i, j}^{\text {new }}<0$, the following volume conserving redistribution of the negative $C_{i, j}^{\text {new }}$ is introduced:

$$
\begin{aligned}
& F_{i, j}^{\mathrm{new}}=0, \quad F_{i-1, j}^{\mathrm{new}}=F_{i-1, j}+\frac{u_{w}}{u_{w}+v_{s}} \frac{r_{i}}{r_{i-1}} C_{i, j}^{\mathrm{new}}, \\
& F_{i, j-1}^{\mathrm{new}}=F_{i, j-1}+\frac{v_{s}}{u_{w}+v_{s}} C_{i, j}^{\mathrm{new}}
\end{aligned}
$$

and if $C_{i, j}^{\text {new }}>1$, then the cell $(i, j)$ is considered as being completely filled and the supplementary amount of melt will be conservatively divided in two directions over the two neighboring E cells

$$
\begin{aligned}
& F_{i, j}^{\mathrm{new}}=1, \quad F_{i+1, j}^{\mathrm{new}}=\frac{u_{e}}{u_{e}+v_{n}} \frac{r_{i}}{r_{i+1}}\left(C_{i, j}^{\mathrm{new}}-1\right), \\
& F_{i, j+1}^{\mathrm{new}}=\frac{v_{n}}{u_{e}+v_{n}}\left(C_{i, j}^{\mathrm{new}}-1\right)
\end{aligned}
$$

The turbulence field quantities are initialized in the two new S cells as in Eq. (15) by $\Phi_{i+1, j}^{\mathrm{new}}=\Phi_{i, j+1}^{\mathrm{new}}=\Phi_{i, j}$.

For the thick line E cell in Fig. 5(b), $C_{i, j}^{\text {new }} \geq 0$ can only result if an inward flow with $Q_{\text {block }} \leq 0$ appears in Eq. (11). In this case, also $F_{i, j}^{\text {new }}=C_{i, j}^{\text {new }}$ and the quantities $\Phi=k, \varepsilon, \mu_{\text {eff }}$ are determined by velocity-averaging using the left and bottom velocities, $u_{l}$ and $v_{b}$, if these have an inward orientation ensured by the "max" feature

$$
\begin{aligned}
& \Phi_{i, j}^{\text {new }}=\frac{u_{l} \Phi_{i-1, j}+v_{b} \Phi_{i, j-1}}{u_{l}+v_{b}}, \\
& u_{l}=\max \left\{u_{w}, 0\right\}, \quad v_{b}=\max \left\{v_{s}, 0\right\}
\end{aligned}
$$

Finally, for a diagonally situated $\mathrm{F}$ cell to an one-cell block, e.g. the cells $(i-1, j-1)$ in the Figs. 5(a) and 5(b), if $F_{i-1, j-1}^{\text {new }}<1$ and $F_{i, j-1}^{\text {new }}>0$ or $F_{i-1, j}^{\text {new }}>0$, then a redistribution using an inward gathering of the melt volume will be introduced. For example, when $F_{i-1, j-1}^{\text {new }}<1$ and $F_{i, j-1}^{\text {new }}>0$, then $S_{F}=F_{i-1, j-1}^{\text {new }}+\frac{r_{i}}{r_{i-1}} F_{i, j-1}^{\text {new }}$ is determined and

$$
\begin{aligned}
& \text { if } S_{F} \leq 1 \text {, then } \quad F_{i-1, j-1}^{\text {new }}=S_{F}, \quad F_{i, j-1}^{\text {new }}=0 \\
& \text { else } \quad F_{i-1, j-1}^{\text {new }}=1, \quad F_{i, j-1}^{\text {new }}=\frac{r_{i-1}}{r_{i}}\left(S_{F}-1\right) .
\end{aligned}
$$

\subsection{Time Step Restrictions}

A free surface portion can be displaced during a time step from an S cell at the most into its adjacent cells. Because the VOF-method and the free surface reconstruction are performed for MC blocks with various configurations, under$\left(F^{\text {new }}<0\right)$ or overshoots $\left(F^{\text {new }}>1\right)$ may occur only if the already mentioned stability limited time step (5) is used. Therefore, limitations of the values $C_{i, j}^{\text {new }}$ have to be introduced; e.g. for the MC block shown in Fig. 4(b), $C_{i, j}^{\text {new }}$ must be restricted according to the Eqs. (13), with $F_{i-1, j}=1$, and (14) between the limits $C_{\min i, j}=-r_{i-1} / r_{i}$ and $C_{\max i, j}=1+r_{i+1} / r_{i}$, respectively. For the vertical MC block in Fig. 4(a), $C_{\min i, j}=-1, C_{\max i, j}=2$ and by imposing all these limits in Eq. (11), the following time steps result

$\Delta t_{1 i, j}=\frac{C_{\min i, j}-F_{i, j}}{C_{i, j}^{\text {new }}-F_{i, j}} \Delta t, \quad \Delta t_{2 i, j}=\frac{C_{\max i, j}-F_{i, j}}{C_{i, j}^{\text {new }}-F_{i, j}} \Delta t$

For the bold circumlined E cell in Fig. 5(b), $C_{i, j}^{\text {new }} \geq 0$, and as a result, $\Delta t_{1 i, j}$ is no more determined for it. Because its velocities $u_{e}=v_{n}=0$ (Fig. 5(b)), the E cell cannot be overfilled and therefore, $\Delta t_{2 i, j}$ is calculated using $C_{\max i, j}=1$. Further, a maximal admissible time step, $\Delta t_{\max }$, is determined for all MC blocks by

$$
\Delta t_{\max }=\min \left\{\Delta t_{1 i, j}, \Delta t_{2 i, j}\right\}
$$

where, "min" terms the smallest value for all MC blocks.

For applying the MC-VOF method, the following steps have to be taken to update the VOF function field. First, all $C^{\text {new }}$-values are calculated by applying Eq. (11) for each MC block. Second, the time step (22) is evaluated, and if $\Delta t_{\max }<$ $\Delta t$, then the whole flow and turbulence fields are recalculated employing the smaller time step $\Delta t_{\max }$. Afterwards, all nearsurface $F$-values are updated to the new time level, e.g. for a levitated melt, firstly for all $\mathrm{E}$ cells situated in the surface concave corners (Fig. 5(b)), secondly for all corner S cells (Fig. 5(a)), further for all MC blocks of the upper MHS, the lower MHS and the MVS and then for the F cells diagonally situated to the one-cell blocks. Finally, the free surface is reconstructed by the SLIC method.

\section{Considerations on the Simulation's Improvements}

\subsection{Improvement of the Pressure Calculation}

The pressure in $\mathrm{S}$ cells is more accurately determined by taking into account also the electromagnetic normal force density $f_{\text {en }}$ exerted on the melt free surface, contained in Eq. (10) in the resulting force density $f_{n}$ (Fig. 3).

Its consideration becomes more important in electromagnetic fields with higher frequencies. For example, in a field with a small penetration depth $\delta \rightarrow 0$, the electromagnetic force densities calculated inside the melt at the inner points of the staggered grid become negligible. As a result, the potential component of the vertical force density is in Eqs. 
(8) given by $f_{\mathrm{FS}}=-\rho g$, where $g$ represents the gravitational acceleration. Thus, if the classical Eq. (9) ${ }^{1)}$ is applied in vertical direction also for $d>h$, then one obtains the interpolated pressure

$$
p_{\mathrm{S}}=p_{\mathrm{s}}+\rho g(d-h)
$$

It follows, that the Lorentz forces are not considered in both the momentum equations discretized for the points of the

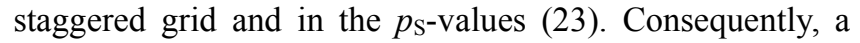
simulation with the classical VOF method and Eq. (9) of the levitation in an electromagnetic field with a high frequency is practically not possible.

If the MFD MC-VOF method is used, then at the melt surface (Fig. 3(b)) $f_{n}=f_{\mathrm{en}}-\rho g$ and in contrast to Eq. (9), Eq. (10) yields:

$$
p_{\mathrm{S}}=p_{\mathrm{s}}+\left(\rho g-f_{\mathrm{en} n}\right)(d-h)
$$

where also the electromagnetic forces are taken into account. Therefore, an inductively levitation can be simulated even for $\delta \rightarrow 0$ by using the Eq. (10) for the pressure calculation in the $\mathrm{S}$ cells.

\subsection{Improvement of the Free Surface Treatment}

The MC-VOF method was established by a generalization and extension to turbulent MFD flows of the VOF-LHF method. To prevent the rounding of the $F$-values, by which the volume conservation is annihilated, and also to avoid the numerical generation of unphysical holes and separated droplets, both methods use an inward fluid gathering after each calculation time step. The effects resulted by using this procedure were detailed presented in the Refs. 5), 6) and 7) by the improved surface reconstruction (i.e. with a reduced deformation and without unphysical holes and droplets) of fluid bodies with different forms translated, revolved or stretched in prescribed uniform, rotational or reversed vortex velocity fields. The improved free surface advection and reconstruction was also illustrated by its strictly volume conserving displacement and by the considerable decreasing of the amount of holes and droplets ('flotsam' and 'jetsam') in the dambreak flow simulation. ${ }^{6,7)}$

Because the effects of the inward gathering procedure were thus detailed demonstrated for the hydrodynamic flows, these effects will be briefly assessed in this paper for the MFD flow in an induction crucible furnace (ICF) described in Table 1 and Fig. 7. $^{21)}$ In the numerical simulation of the transient MFD field at inductor feeding, the inductor was considered to be supplied at the time $t \geq 0$ with a sinusoidal current of constant r.m.s. value, $I$, given in Table 1. Because of the holes numerically generated in the

Table 1. Characteristics of the laboratory ICF (Fig. 7). ${ }^{21)}$

\begin{tabular}{lc}
\hline frequency, r.m.s. value of the inductor current $(I)$ & $386 \mathrm{~Hz}, 2 \mathrm{kA}$ \\
number of inductor series turns & 11 \\
inferior $(a)$, superior $(b)$ heights of the inductor ends & $47.5 \mathrm{~mm}, 570 \mathrm{~mm}$ \\
inner diameter of inductor, of crucible & $385 \mathrm{~mm}, 316 \mathrm{~mm}$ \\
height of Wood's metal melt (for $I=0)$ & $400 \mathrm{~mm}$ \\
electrical conductivity $(\sigma)$, density $(\rho)$, & $1 \mathrm{MS} / \mathrm{m}, 9.4 \mathrm{~g} / \mathrm{cm}^{3}$, \\
surface tension $(\gamma)$ of Wood's metal & $0.46 \mathrm{~N} / \mathrm{m}$ \\
\hline
\end{tabular}

fluid by applying the classical Hirt-Nichols' Volume of Fluid (HN-VOF) method ${ }^{1,5,6)}$ in cylindrical coordinates, ${ }^{22)}$ the calculated free surface of the melt is wrongly lifted in the ICF. The number and volumes of holes rise at the calculation time advancing and therefore, the mean height of the free surface is continuously increased by the HN-VOF method (Fig. 8). In contrast, the applying of the MC-VOF method yields a constant transient mean melt height shown in Fig. 8. Due to the rounding of the $F$-values determined with the donor-acceptor scheme when using the HN-VOF method, ${ }^{1)}$ the difference of the final and initial volumes at $t=$ $20 \mathrm{~s}$ and $t=0 \mathrm{~s}$, respectively, is after 8000 calculation time steps equal to $186.7 \mathrm{~cm}^{3}$, which is hundred times greater than the absolute value of the volume difference $1.8 \mathrm{~cm}^{3}$ obtained with the MC-VOF method, the latter value representing $0.0057 \%$ of the whole melt volume in the ICF.

The experimental verification of the free surface profile calculated with the MFD MC-VOF method in the described test ICF will be presented in the second part of this paper.

\section{Conclusions}

In this first part of a two-part paper, an MC-version of the VOF method has been developed for the free surface calculation of turbulent MFD flows, with the following simulation's improvements:

(1) Simple computation of the electromagnetic, force,

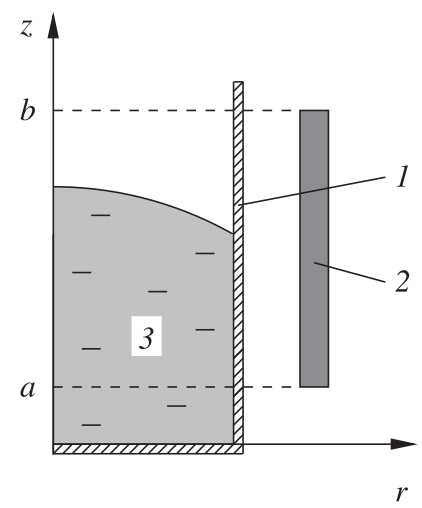

Fig. 7. Cross section of test furnace (schematic): 1 , nonmagnetic steel crucible; 2 , inductor; 3 , melt.

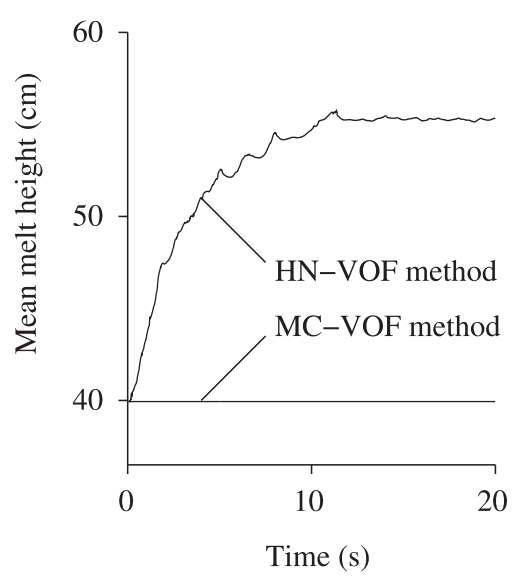

Fig. 8. Calculated transient mean height of the free surface in the ICF. 
and turbulence fields in the S cells considered, as in the flow simulations with the VOF-LHF method, ${ }^{3,5-7)}$ to be completely filled with fluid. As a result, the complicate procedures employed in the Refs. 4 and 8-10) to update after each computational time step the physical properties in the surface cells are avoided.

(2) Development of a model for turbulent MFD flows by using a realistic determination of the convective and production terms in the transport equations of $k$ and $\varepsilon$, i.e. with different values for the SE velocities, while the hydrodynamic and MFD models in 3-10) only for laminar flows were established.

(3) More accurately calculation of the pressure field by taking into account also the normal Lorentz forces exerted on the outer surfaces of the S cells, which are not used in the discretized momentum equations.

(4) Rigorous volume conserving displacement of the free surface by applying the VOF method to non-overlapping MC blocks and calculation stability by using of precisely determined time steps guaranteeing that a surface portion contained in an $\mathrm{S}$ cell can pass at the most the boundaries of the respective $\mathrm{S}$ cell. Thus, the rounding of $F$-values used in $1,9,10$ ) is avoided, the possibility of multiple fluid fluxing, which can occur when using the VOF-LHF method, ${ }^{3,5-7)}$ is eliminated and, in contrast to the VOF-LHF method, the $\mathrm{MC}-\mathrm{VOF}$ method can be easily applied also in cylindrical coordinates.

(5) Free surface inward gathered reconstruction avoiding the creation of unphysical holes and droplets. The surface is precisely reconstructed and therefore, the $F$-values don't need to be corrected after each time step by an antidiffusion procedure ${ }^{8)}$ or a supplementary advection, ${ }^{4)}$ respectively, and also a non-linear treatment of the physical properties in the surface cells, ${ }^{9,10)}$ hindering the mass con- servation is not needed.

The experimental validation of the MFD MC-VOF method by comparison of the calculated results with the measurements in three laboratory setups will be presented in the second part of this two-part paper.

\section{REFERENCES}

1) C. W. Hirt and B. D. Nichols: J. Comp. Phys., 39 (1981), 201

2) M. Rudman: Int. J. Numer. Meth. Fluids, 24 (1997), 671.

3) Numerical Methods for Fluid Dynamics VI, ed. by M. J. Baines, ICFD, Oxford, (1998), 493.

4) P. Liovic, J.-L. Liow and M. Rudman: ISIJ Int., 41 (2001), 225.

5) J. Gerrits: Ph. D. Thesis, University of Groningen, (2001).

6) K. M. T. Kleefsman: Ph. D. Thesis, University of Groningen, (2005).

7) K. M. T. Kleefsman, G. Fekken, A. E. P. Veldman, B. Iwanowski and B. Buchner: J. Comp. Phys., 206 (2005), 363.

8) K. Takatani: ISIJ Int., 47 (2007), 545.

9) T. Tagawa: ISIJ Int., 47 (2007), 574.

10) Y. Shibasaki, K. Ueno and T. Tagawa: ISIJ Int., 50 (2010), 363.

11) O. Peşteanu: Proc. on USB-Flash of 54th Int. Scientific Colloquium, Ilmenau University of Technology, Ilmenau, (2009), Session 7.2: Electromagnetic processing of materials.

12) H. L. Huang, A. Ying and M. A. Abdou: Fusion Engineering and Design, 63-64 (2002), 361.

13) O. Peşteanu, E. Baake and B. Nacke: Proc. of Workshop Elektroprozesstechnik, TU Ilmenau, Ilmenau, (2004), Rep. 8.

14) O. Peşteanu and K. Schwerdtfeger: Proc. on CD-ROM of 48th Int. Scientific Colloquium, Ilmenau University of Technology, Ilmenau, (2003), Topic 9 Power Electrical Engineering, 9.6 Electro-Processing Technol.

15) O. Peșteanu and K. Schwerdtfeger: ISIJ Int., 43 (2003), 1556.

16) Handbook of numerical heat transfer, ed. by W. J. Minkowycz, E. M. Sparrow, G. E. Schneider and R. H. Pletcher, John Wiley \& Sons, New York, (1988), 283, 350.

17) U. Brockmeier: Dissertation, Ruhr Universität Bochum, (1987).

18) O. Peşteanu: Simulation of Turbulent Molten Metal Flow in Electromagnetic Field, VDI, Düsseldorf, (1994), 7.

19) Magnetohydrodynamics in Process Metallurgy, ed. by J. Szekely, J. W. Evans, K. Blazek and N. El-Kaddah, TMS Publication, Warrendale, PA, (1991), 21.

20) T. Griebel, T. Dornseifer and T. Neunhoeffer: Numerische Simulation in der Strömungsmechanik, Vieweg, Braunschweig, (1995), 92.

21) M. Kirpo: Ph. D. Thesis, University of Latvia, Riga, (2008).

22) J. R. Richards: Dissertation, University of Delaware, (1994). 